
\title{
Model Calculation for the Field Enhancement Factor of Carbon Nanowall Array
}

Tomohiko YAMAKAMI' ${ }^{\dagger}$, Masahiro YAMASHITA ${ }^{\dagger}$, Rinpei HAYASHIBE ${ }^{\dagger}$, Nonmembers, $^{\dagger}$ and Kiichi KAMIMURA ${ }^{\dagger a}$, Member

\begin{abstract}
SUMMARY To estimate the field emission current associated with an array of carbon nanowalls (CNWs), the model of the floating rods between anode and cathode plates was proposed. An approximate formula for the enhancement factor was derived, showing that the interwall distance of the $\mathrm{CNW}$ array critically affects the field emission. The field enhancement factor was almost one order of magnitude less than that of vertically aligned CNTs. Considering the field emission current density, the field emission can be optimized when the interwall distance is comparable with the wall height. For same separation distance, the macroscopic field strength of the CNW array is almost one order of magnitude higher than that of vertical CNT array to obtain the emission current of $1 \mathrm{~mA}$ from the cathode surface of $1 \mathrm{~cm}^{2}$.

key words: field emission, field enhancement factor, nanowall
\end{abstract}

\section{Introduction}

An important factor in field emission is the relationship between the applied and the local electric field where electron tunneling occurs. When a voltage is applied across a carbon nanowall (CNW) and a perpendicular planar anode, the nanoscale width and the high ratio lead to a much greater local field at the top of CNW.

Simulation and calculation for the field emission from carbon nanotubes (CNTs) are mainly concentrated on the vertically aligned CNTs. Wang et al proposed the floating sphere model to calculate the apex field-enhancement factor for an individual CNT, leading to a simple formula for the factor [1]. They also reported the field-enhancement factor for vertically aligned CNT array [3].

The enhancement factor of CNT arrays should be smaller than that of an individual CNT due to the electrostatic interactions between CNTs. And many experiments had shown that the intertube distance critically affects the field-enhancement factor of CNT array, and that the field emission could be optimized when the intertube distance is comparable with the tube height.

Few papers have reported about field distribution around vertically aligned CNWs. To estimate the field emission current associated with the CNW array, the model of the floating rods between anode and cathode plates was proposed. An approximate formula for the enhancement factor was derived, showing that the interwall distance of the $\mathrm{CNW}$ array critically affects the field emission.

\footnotetext{
Manuscript received April 22, 2011.

$\dagger$ The authors are with the Faculty of Engineering, Shinshu University, Nagano-shi, 380-8553 Japan.

a) E-mail: kamimur@shinshu-u.ac.jp

DOI: $10.1587 /$ transele.E94.C.1867
}

\section{Model and Calculation}

2.1 Floating Rod Model for the Field Enhancement Factor of the Nanowall Array

The model shown in Fig. 1 was used to calculate the field enhancement factor for the vertically aligned CNWs on the cathode. As shown in Fig. 1, the CNWs stand perpendicularly on the cathode plane, having a plate shape of the height $h$ and closed at the top with a cylindrical cap with the radius $\rho$. Since we assumed the $\mathrm{CNW}$ is conducting, the cathode potential is maintained zero over whole the surface of CNWs. Owning to the macroscopic electric field, almost induced charges are exiting at and near the top of CNWs. Thus, the rod with the radius $\rho$ at the height of $h$ can be electrically replaced with the CNW.

\subsection{Floating Charge and Dipole Model}

The electrically grounded rods placed at height $h$ can be replaced with the linear arrayed charge of $-q$ and linear arrayed dipole $-p$, together with image charge $q$ and image dipole $p$. The field produced by the images must satisfy the boundary conditions at the conductor surfaces and the cathode plate.

The electric potential $V(r)$ at the distance $r$ from a straight line charge with infinite length in free space as shown in Fig. 2(a) is,

$$
V(r)-V\left(r_{0}\right)=\frac{q}{2 \pi \epsilon_{0}} \ln \left(\frac{r_{0}}{r}\right)
$$

where $q$ is the charge per unit length, $\epsilon_{0}$ is premitivity of free space and $V\left(r_{0}\right)$ is the potential at $r=r_{0}$.

The electric potential $V(r, \phi)$ around a linearly arrayed dipole with infinite length as shown in Fig. 2(b) is,

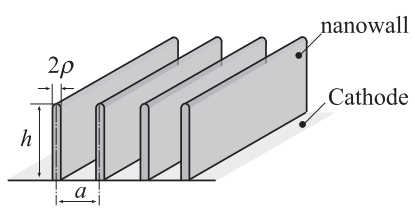

(a) arrayed nanowalls

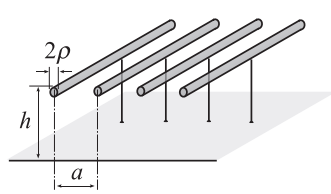

(b) floating rods model for nanowalls
Fig. 1 The schematic of the nanowall array and its floating rods model. 


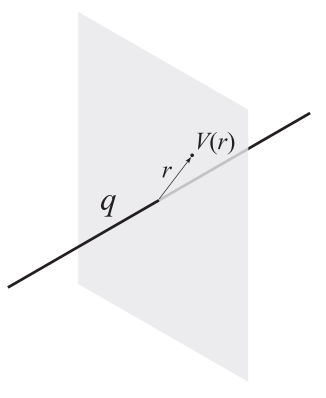

(a) linear arrayed charge

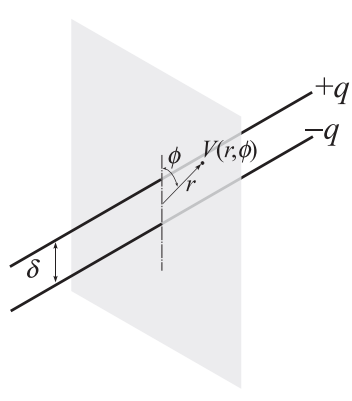

(b) linear arrayed dipole
Fig. 2 Linear charge and linearly arrayed dipole.

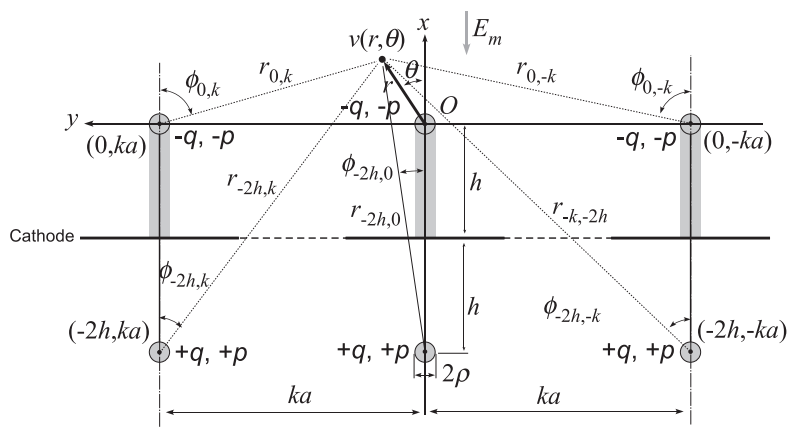

Fig. 3 Linear charge and linearly arrayed dipole model for calculation of potential distribution around carbon nanowall.

$$
\begin{aligned}
V(r, \phi)- & V\left(r_{0}, \phi_{0}\right)= \\
& \frac{q}{2 \pi \epsilon_{0}} \ln \left(\frac{r_{0}}{\sqrt{r^{2}-r \delta \cos \phi+\delta^{2} / 4}}\right) \\
& -\frac{q}{2 \pi \epsilon_{0}} \ln \left(\frac{r_{0}}{\sqrt{r^{2}+r \delta \cos \phi+\delta^{2} / 4}}\right) \\
\cong & \frac{q}{4 \pi \epsilon_{0}}\left\{\ln \left(1+\frac{\delta}{r} \cos \phi\right)-\ln \left(1-\frac{\delta}{r} \cos \phi\right)\right\} \\
\cong & \frac{q \delta}{2 \pi \epsilon_{0} r} \cos \phi=\frac{p}{2 \pi \epsilon_{0} r} \cos \phi
\end{aligned}
$$

where $\phi$ is the angle between $r$ and the direction from $-q$ to $+q, \delta$ is distance between the linear charges, $p \equiv q \delta$ is dipole moment per unit length and $V\left(r_{0}, \phi_{0}\right)$ is the potential at $r=r_{0}$ and $\phi=\phi_{0}$. The value of $\delta$ is assumed to be much smaller than $r$ or $r_{0}$ and $\ln (1+x) \cong x$ for $x \ll 1$.

Figure 3 shows the model for the nanowall array. The nanowalls are placed at $y= \pm k a$, where $a$ is the spacing between nanowalls and $k$ is integer. The height of the nanowall is $h$, the width of it is $2 \rho$ and the length of it is assumed to be infinity. The top of the nanowall is assumed to be cylindrical.

In order to estimate the field strength near the top of the nanowall, we propose a simple model of floated linear charges and dipoles between parallel anode and cathode plates. Take the cylindrical center of the cylindrical coordinate as the origin $O$, and the vertical axis through $O$ as the $x$ axis. The $y z$ plane located at $x=-h$ denotes the cathode plate.

In our model of floating linear line charges and dipoles combined with the image method, the potential near a cylindrical floating rods (the upper half of the cylindrical rod coincides with the cap of a nanowall) with its center at the origin $O$ is considered as produced by (a) the two layers of linear-charge arrays each point with charge $-q$ or $q$ at symmetrically situated above and below the cathode plane as shown partially in Fig. 3, (b) the two layers of linear arrayed dipoles each with a dipole moment $-p$ or $p$, locating at each linear charge and pointing downwards, and (c) the applied voltage $V$ on the anode and cathode planes causing a mean field $-E_{m}$ between them. Linear charges and dipoles of infinite length were arrayed to establish zero potential $(V(\rho)=0)$ at the distance $\rho$ from the charges os dipoles.

When the potential reference point is selected on the cathode and $V\left(r_{0}\right)=V\left(r_{0}, \phi_{0}\right)=0$, the electric potential at the point $(r, \theta)$ to the linear charges and linear arrayed dipoles is,

$$
\begin{aligned}
V(r, \theta)= & E_{m}(l+r \cos \theta)+\frac{-q}{2 \pi \epsilon_{0}} \ln \left(\frac{r_{0}}{r}\right)+\frac{-p}{2 \pi \epsilon_{0} r} \cos \theta \\
& +\frac{q}{2 \pi \epsilon_{0}} \ln \left(\frac{r_{0}}{r_{-2 h, 0}}\right)+\frac{p}{2 \pi \epsilon_{0} r_{-2 h, 0}} \cos \phi_{-2 h, 0} \\
+ & \sum_{k=1}^{\infty}\left\{\frac{-q}{2 \pi \epsilon_{0}} \ln \left(\frac{r_{0}}{r_{0, k}}\right)+\frac{-p}{2 \pi \epsilon_{0} r_{0, k}} \cos \phi_{0, k}\right. \\
& \frac{-q}{2 \pi \epsilon_{0}} \ln \left(\frac{r_{0}}{r_{0,-k}}\right)+\frac{-p}{2 \pi \epsilon_{0} r_{0,-k}} \cos \phi_{0,-k} \\
& \frac{q}{2 \pi \epsilon_{0}} \ln \left(\frac{r_{0}}{r_{-2 h, k}}\right)+\frac{p}{2 \pi \epsilon_{0} r_{-2 h, k}} \cos \phi_{-2 h, k} \\
& \left.\frac{q}{2 \pi \epsilon_{0}} \ln \left(\frac{r_{0}}{r_{-2 h,-k}}\right)+\frac{p}{2 \pi \epsilon_{0} r_{-2 h,-k}} \cos \phi_{-2 h,-k}\right\}
\end{aligned}
$$

where,

$$
\begin{aligned}
r_{-2 h, 0} & =\sqrt{(2 h+r \cos \theta)^{2}+(r \sin \theta)^{2}} \\
r_{0, \pm k} & =\sqrt{(r \cos \theta)^{2}+(a k \mp r \sin \theta)^{2}} \\
r_{-2 h, \pm k} & =\sqrt{(2 h+r \cos \theta)^{2}+(k a \mp r \sin \theta)^{2}} \\
\cos \phi_{-2 h, 0} & =(2 h+r \cos \theta) / r_{-2 h, 0} \\
\cos \phi_{0, \pm k} & =(r \cos \theta) / r_{ \pm k, 0} \\
\cos \phi_{-2 h, \pm k} & =(2 h+r \cos \theta) / r_{ \pm k,-2 h}
\end{aligned}
$$

In the case of $r \ll h, a$ and $r_{0}, V(r, \theta)$ can be approximated by,

$$
\begin{aligned}
V(r, \theta) \cong & E_{m}(h+r \cos \theta)+\frac{-q}{2 \pi \epsilon_{0}}\left\{\ln \left(\frac{2 h}{r}\right)+\frac{r}{2 h} \cos \theta\right\} \\
& +\frac{-p}{2 \pi \epsilon_{0} r} \cos \theta+\frac{p}{4 \pi \epsilon_{0} h}+\frac{-q}{2 \pi \epsilon_{0}} S_{1} \\
& +\frac{-p}{2 \pi \epsilon_{0}} \frac{2 r \cos \theta}{a^{2}} S_{2}+\frac{p}{2 \pi \epsilon_{0}} \frac{4 h}{a^{2}} S_{3}
\end{aligned}
$$

where 


$$
\begin{aligned}
& S_{1}=\sum_{k=1}^{\infty} \ln \left(1+\frac{4 h^{2}}{a^{2}} \frac{1}{k^{2}}\right) \\
& S_{2}=\sum_{k=1}^{\infty}\left(\frac{1}{k^{2}}-\frac{1}{k^{2}+\frac{4 h^{2}}{a^{2}}}\right) \\
& S_{3}=\sum_{k=1}^{\infty}\left(\frac{1}{k^{2}+\frac{4 h^{2}}{a^{2}}}\right)
\end{aligned}
$$

Because the nanowalls are electrically connected to the cathode plate, the potential at $r=\rho$ must be zero, so,

$$
\begin{aligned}
V(\rho, \theta)= & E_{m}(h+\rho \cos \theta)+\frac{-q}{2 \pi \epsilon_{0}}\left\{\ln \left(\frac{2 h}{\rho}\right)+\frac{\rho}{2 h} \cos \theta\right\} \\
& +\frac{-p}{2 \pi \epsilon_{0} \rho} \cos \theta+\frac{p}{4 \pi \epsilon_{0} h}+\frac{-q}{2 \pi \epsilon_{0}} S_{1} \\
& +\frac{-p}{2 \pi \epsilon_{0}} \frac{2 \rho \cos \theta}{a^{2}} S_{2}+\frac{p}{2 \pi \epsilon_{0}} \frac{4 h}{a^{2}} S_{3}=0
\end{aligned}
$$

The Eq. (14) must be valid for all $\theta$, so $q$ and $p$ must be satisfy following equations,

$$
\begin{array}{r}
E_{m} h+\frac{-q}{2 \pi \epsilon_{0}} \ln \left(\frac{2 h}{\rho}\right)+\frac{p}{4 \pi \epsilon_{0} h}+\frac{-q}{2 \pi \epsilon_{0}} S_{1} \\
+\frac{p}{2 \pi \epsilon_{0}} \frac{4 h}{a^{2}} S_{3}=0 \\
E_{m} \rho+\frac{-q}{4 \pi \epsilon_{0}} \frac{\rho}{h}+\frac{-p}{2 \pi \epsilon_{0} \rho}+\frac{-p}{2 \pi \epsilon_{0}} \frac{2 \rho}{a^{2}} S_{2}=0
\end{array}
$$

so the values of $p$ and $q$ are,

$$
\begin{aligned}
p & =\frac{2 \pi \epsilon_{0} \rho^{2} E_{m}}{2+\frac{2 \rho^{2}}{a^{2}} S_{2}}\{1 \\
& \left.-\frac{1}{2 h} \frac{h\left(1+\frac{2 \rho^{2}}{a^{2}}\right)+\rho^{2}\left(\frac{1}{2 h}+\frac{4 h}{a^{2}} S_{3}\right)}{\left(\ln \frac{2 h}{\rho}+S_{1}\right)\left(1+\frac{2 \rho^{2}}{a^{2}} S_{2}\right)+\frac{\rho^{2}}{2 h}\left(\frac{1}{2 h}+\frac{4 h}{a^{2}} S_{3}\right)}\right\} \\
q & =2 \pi \epsilon_{0} E_{m} \frac{h\left(1+\frac{2 \rho^{2}}{a^{2}} S_{2}\right)+\rho\left(\frac{\rho}{2 h}+\frac{2 \rho^{2}}{a^{2}} S_{3}\right)}{\left(\ln \frac{2 h}{\rho}+S_{1}\right)\left(1+\frac{2 \rho^{2}}{a^{2}} S_{2}\right)+\left(\frac{\rho^{2}}{4 h^{2}}+\frac{4 \rho^{2}}{a^{2}} S_{3}\right)}
\end{aligned}
$$
is,

The electric field strength $\left(E_{\rho}\right)$ at the top of the nanwall

$$
E_{\rho}=-\left.\frac{d V(r, \theta)}{d r}\right|_{r=\rho, \theta=0}
$$

$$
\begin{gathered}
=-E_{m}\left(1+\frac{1}{1+\frac{2 \rho^{2}}{a^{2}} S_{2}}\right) \\
-E_{m} \frac{h\left(1+\frac{2 \rho^{2}}{a^{2}}\right)+\rho^{2}\left(\frac{1}{2 l}+\frac{4 h}{a^{2}} S_{3}\right)}{\left(\ln \frac{2 h}{\rho}+S_{1}\right)\left(1+\frac{2 \rho^{2}}{a^{2}} S_{2}\right)+\left(\frac{\rho^{2}}{4 h^{2}}+\frac{4 \rho^{2}}{a^{2}} S_{3}\right)} \\
\times\left\{\frac{1}{\rho}\left(1-\frac{\rho}{2 h}\right)-\frac{1}{2 h} \frac{1}{1+\frac{2 \rho^{2}}{a^{2}} S_{2}}\right\} \\
\cong-2 E_{m}-E_{m} \frac{\frac{h}{\rho}-1}{\ln \frac{2 h}{\rho}+S_{1}}
\end{gathered}
$$

where $\rho$ is assumed to be much smaller than $h$ and $a$. The field enhancement factor $\beta$ is,

$$
\begin{aligned}
\beta & =\left|\frac{E_{\rho}}{E_{m}}\right|=\frac{\frac{h}{\rho}-1}{\ln \frac{2 h}{\rho}+S_{1}}+2 \\
& =\frac{\frac{h}{\rho}-1}{\ln \frac{2 h}{\rho}+\sum_{k=1}^{\infty} \ln \left(1+\frac{4 h^{2}}{a^{2}} \frac{1}{k^{2}}\right)}+2
\end{aligned}
$$

The $S_{1}$ can not be expressed in a simple formula. The value of $\beta$ can be obtained by calculating the value of $S_{1}$ numerically.

\section{The Influence of the Intertube Distance}

\subsection{Field Enhancement Factor $\beta$}

Figure 4 shows the dependence of field enhancement factor of CNW array on the normalized interwall distance $(a / h)$. The field enhancement factor of vertically aligned CNTs was also calculated using the equation derived by Wang et al. [3], and shown in Fig. 4. Wang et al. [3] reported the field enhancement factor $\beta$ of vertically aligned CNTs by the same method described above. The value of $\beta$ is,

$$
\begin{aligned}
& \beta=\frac{h}{\rho}\left(\frac{1}{1+Y}\right)+\frac{1}{2}\left(\frac{1}{1+Y}\right)^{2}+3 \\
& Y \equiv \frac{\rho}{R} \sum_{n} \sum_{m}\left(\frac{4}{\sqrt{n^{2}+m^{2}}}-\frac{4}{\sqrt{n^{2}+m^{2}+\frac{4 h^{2}}{a R^{2}}}}\right)
\end{aligned}
$$

where $h$ and $\rho$ are the length and diameter of CNT, respectively, and $R$ is intertuve distance [3]. Equation (20) was used to calculate the data for the CNW array. In Fig. 4 the 


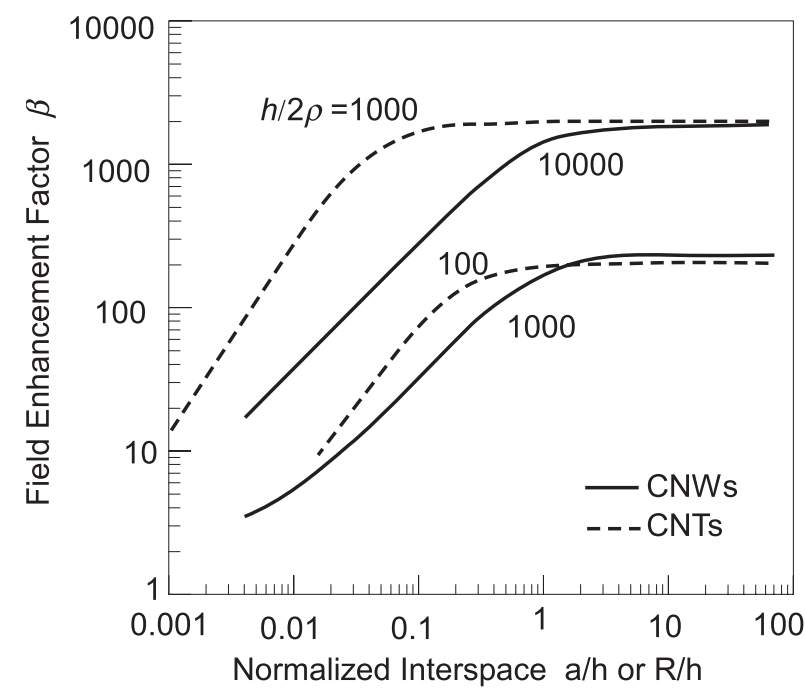

Fig. 4 Field enhancement factor of CNW array and vertically aligned CNTs against the normalized interspace.

dashed lines indicate the data for vertical aligned CNT array and solid lines are for the CNW array. The sums $\left(\sum\right)$ in Eqs. (20) and (21) were calculated from $n=1, m=0$ or $k=1$ to $m=n=k=1000$.

When the distance is less than the height of CNWs $(a / h<1)$, the enhancement factor for the CNW array decreases rapidly with decreasing distance. The decrease of field enhancement factor for the CNW array starts at higher $a / h$ value than that for vertical CNT array.

\subsection{Field Emission Current Density}

The emission current from cathode is proportional to the area of emission site. The emission area of the CNW is thought to be wider than that of vertical CNT. So the emission current must be compared to evaluate the performance as the field emission cathode.

The Fowler-Nordheim equation for field emission current density $J$ in $\mathrm{A} / \mathrm{cm}^{2}$ is,

$$
J=1.54 \times 10^{-6} \frac{E_{x}^{2}}{\phi_{m}} \exp \left\{-6.83 \times 10^{7} \frac{\phi_{m}^{3 / 2}}{E_{x}}\right\}
$$

where, $\phi$ is work function in $\mathrm{eV}$ and $E_{x}$ is electric field in $\mathrm{V} / \mathrm{cm}[4]-[7]$.

The emission current from cathode surface of $1 \mathrm{~cm} \times$ $1 \mathrm{~cm}$ was estimated from Eq. (23) and the values of $\beta$ shown in Fig. 4. The emission area of individual CNW and individual CNT are assumed to be $2 \rho \times 1 \mathrm{~cm}$ and $\pi \rho^{2}$, respectively. There are $1 / a \mathrm{CNWs}$ or $1 / R^{2} \mathrm{CNTs}$ on the cathode surface of $1 \times 1 \mathrm{~cm}^{2}$. The total emission area per $1 \mathrm{~cm}^{2}$ of the cathode for CNW array $\left(A_{W}\right)$ and that for vertical CNT array $\left(A_{T}\right)$ are,

$$
\begin{aligned}
& A_{W}=2 \rho \frac{1}{a}=\frac{2 \rho}{h} \frac{h}{a} \\
& A_{T}=\pi \rho^{2} \frac{1}{R^{2}}=\frac{\pi}{4}\left(\frac{2 \rho}{h}\right)^{2}\left(\frac{h}{R}\right)^{2}
\end{aligned}
$$

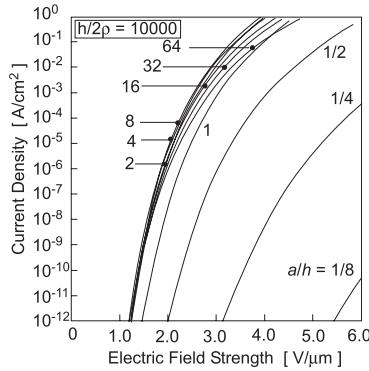

(a) Emission current from arrayed CNWs

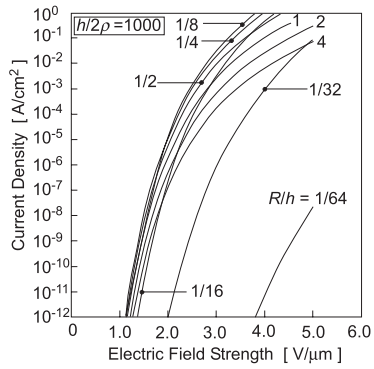

(b) Emission current from arrayed CNTs
Fig. 5 Emission current from arrayed CNWs of $h / 2 \rho=10000$ (a) and that from arrayed CNTs of $h / 2 \rho=1000$ (b).

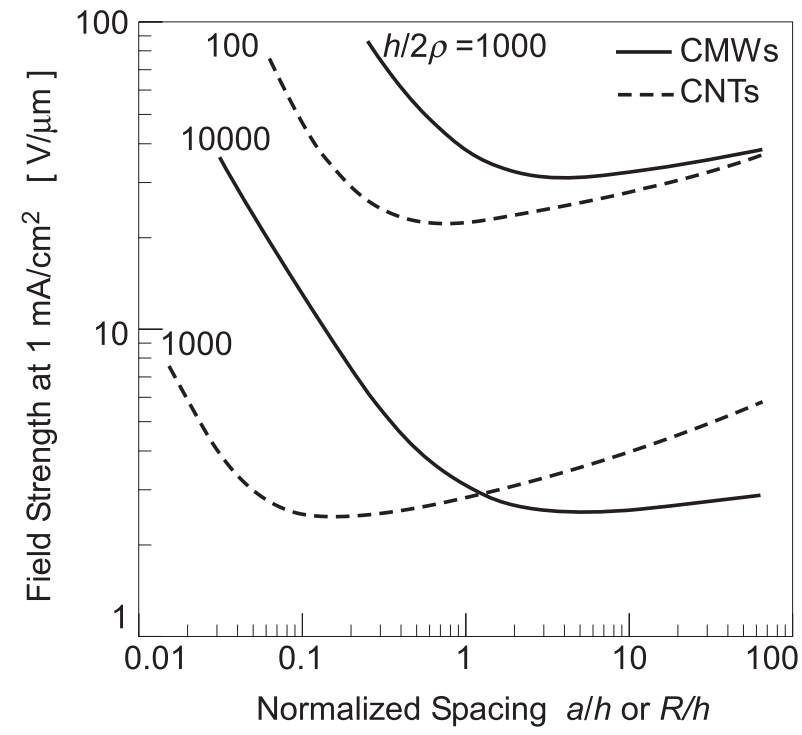

Fig. 6 Macroscopic electric field $E_{m}=V / d$, when the field emission current density is $1 \mathrm{~mA} / \mathrm{cm}^{2}$.

where $a, R$ and $\rho$ are in $\mathrm{cm}$. The work function $\phi$ is assumed to be $5 \mathrm{eV}$. The emission current from the unit cathode area $\left(A_{W} J\right.$ or $\left.A_{T} J\right)$ was calculated from Eq. (23) and Eq. (24) or (25), and shown in Fig. 5. In this calculation, the value of $h / 2 \rho$ was selected to obtain the lowest turn on field of $1-$ $2 \mathrm{~V} / \mu \mathrm{m}$.

The shielding effect seems more serious for arrayed CNWs than for arrayed CNTs. To discuss the dependence of emission current on the interdistance, the field strength to obtain the current density of $1 \mathrm{~mA} / \mathrm{cm}^{2}$ on the cathode surface was calculated. Figure 6 shows the macroscopic electric field $E_{m}=V / d$, when the field emission current density is $1 \mathrm{~mA} / \mathrm{cm}^{2}$ on the cathode surface.

For same $a / h$ or $R / h$ value, although the total emission area of CNW array for the same cathode area is wider than that of vertical CNT array, the macroscopic field strength of CNW array is almost one order of magnitude higher than that of vertical CNT array. The field emission performance seemed to be determined mainly by field enhancement factor $\beta$. 


\section{Conclusion}

The model of the floating lines between anode and cathode plates was used to estimate the field emission current associated with the arrayed of CNWs. When the distance is less than the height of CNWs $(a / h<1)$, the field enhancement factor for the CNW array decreases rapidly with decreasing distance. The decrease of field enhancement factor for CNW array starts at higher $a / h$ value than that for vertical CNT array and is significant in the region of $a / h<1$.

For same inter space to height ratio $(a / h$ or $R / h)$, the macroscopic field strength of CNW array is almost one order of magnitude higher than that of vertical CNT array to obtain the emission current density of $1 \mathrm{~mA} / \mathrm{cm}^{2}$ on the cathode surface. The field emission performance seemed to be determined mainly by field enhancement factor $\beta$.

\section{Acknowledgment}

A part of this research was supported by Regional Innovation Cluster Program of Nagano, granted by MEXT, Japan.

\section{References}

[1] X.Q. Wang, M. Wang, P.M. He, Y.B. Xu, and Z.H. Li, "Model calculation for the field enhancement factor of carbon nanotube," J. Appl. Phys., vol.96, 6752, 2004.

[2] K. Kamimura and T. Yamakami, "Effect of series resistance on field emission current," Jpn J. Appl. Physics, vol.48, 098006, 2009.

[3] M. Wang, Z.H. Li, X.F. Shang, X.Q. Wang, and Y.B. Xu, "Fieldenhancement factor for carbon nanotube array," J. Appl. Phys., vol.98, 014315, 2005.

[4] L.S. Altman, P.V. Pikhitsa, and M. Choi, "Electron field emission from nanacarbons; A two-process model," Appl. Phys. Lett., vol.84, pp.1126-1128, 2004.

[5] R.G. Forbes, "Simple good approximations for the special elliptic functions in standard Fowler-Nordheim tunnering theory for a Schottky-Nordheim barrier," App. Phys. Lett., vol.89, 113112, 2006.

[6] H.C. Miller, "Charge in field intensification factor $\beta$ of an electrode projection (whisker) at short gap length," J. Appl. Phys., vol.38, pp.4501-4504, 1067.

[7] J.T.L. Thong, C.H. Oon, W.K. Eng, W.D. Zhang, and L.M. Gan, "High-current field emission from a vertically aligned carbon nanotube field emitter array," Appl. Phys. Lett., vol.79, pp.2811-2813, 2001.

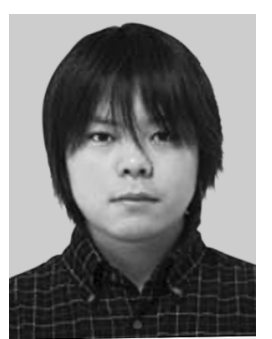

Masahiro Yamashita graduated from Department of Electrical and Electronic Engineering, Shinshu University. He is now a student of Graduate School of Science and Technology, Shinshu University. He has been engaged in research on the the field emission from CNT composite.

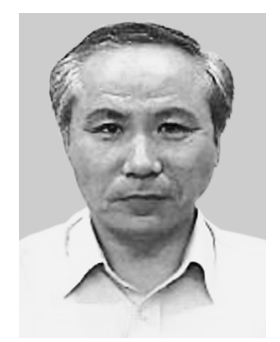

Rinpei Hayashibe graduated from Nagano Technical College in 1969. In 1971, he joined the Faculty of Engineering, Shinshu University. He received his Ph.D. degree in electrical and electronic engineering from Shinshu University, Nagano, Japan, in 2002. His research interests are in the areas of electronic devices, circuits systems.

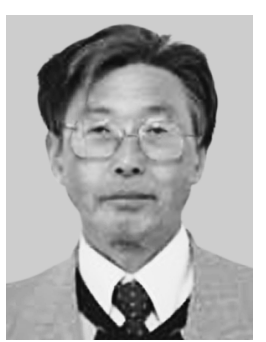

Kiichi Kamimura received the $\mathrm{Ph}$. D degree from Tokyo Institute of Technology, Tokyo, Japan, in 1977. In 1977, he joined Tokyo Institute of Polytechnics, Atsugi, Japan. In 1983, he joined the Department of Electric Engineering, Shinshu University where, since 1999 , he was Professor. He has been engaged in research on the preparation and characterization of interface between compound semiconductor and insulator. He is a member of IEEE and JSAP.

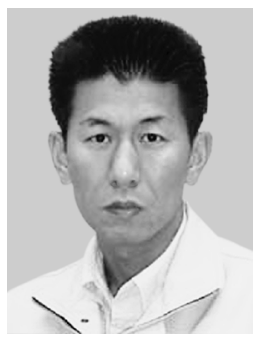

Tomohiko Yamakami joined Shinshu University, Nagano, Japan, in 1985 . He is currently a technical specialist at Shinshu University. He is a member of the Japanese Society of Microscopy. 